\title{
A truly active pseudokinase
}

Cell 175, 1-13 (2018)
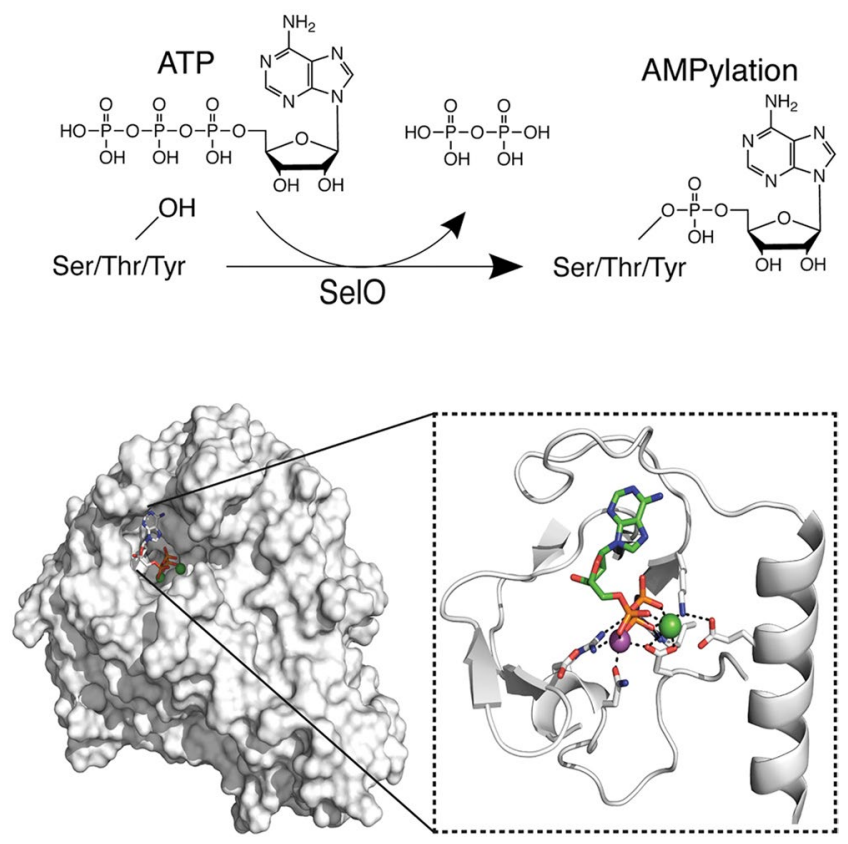

Kinases are enzymes that transfer phosphate groups. However, ten per cent of human protein kinases are classified as pseudokinases, and are believed to be catalytically inactive due to the lack of conserved residues involved in kinase-mediated catalysis and adenosine triphosphate (ATP) binding.

Now, Vincent S. Tagliabracci and co-workers have discovered that selenoprotein-O (SelO) pseudokinases are unexpectedly active enzymes. Specifically, they report that SelO transfers adenosine monophosphate (AMP) to serine, threonine and tyrosine residues on protein substrates - and that this function of SelO plays an important role in the cellular response to oxidative stress.

The idea that SelO might have AMPylation activity (covalent attachment of AMP to protein side chains) arose from analysis of the solved crystal structure of the SelO homolog bound to an ATP derivative from the Gram-negative plant pathogen Pseudomonas syringae. This revealed that SelO adopts a protein kinase-like fold, but ATP is flipped in the active site when compared with common protein kinases. This flip provides a structural and catalytic basis to transfer AMP instead of a phosphate group to protein substrates.

The authors showed that SelO AMPylates proteins involved in redox biology, thereby regulating global S-glutathionylation levels. S-glutathionylation is a reversible mechanism that protects protein thiols from irreversible oxidation. Further experiments on yeast showed that the presence of SelO leads to higher cellular resistance towards oxidative stress. Interestingly, the authors showed that the activity of SelO itself is redox-regulated by an intramolecular disulfide bond formed in oxidizing environments.

SelO is a highly conserved pseudokinase found in plants, bacteria, yeast and mammals, including humans. It can therefore be expected that the previously unrecognized catalytic activity of SelO reported here is a universal mechanism in redox biology and that AMPylation may be more widespread than previously anticipated. This new information will surely motivate follow-up studies to investigate the catalytic activity of this enzyme and its involvement in biological processes in more detail, and to search for catalytic activities of other proteins currently classified as pseudokinases.

\section{Jan-Stefan Völler}

Published online: 9 November 2018 https://doi.org/10.1038/s41929-018-0186-2 